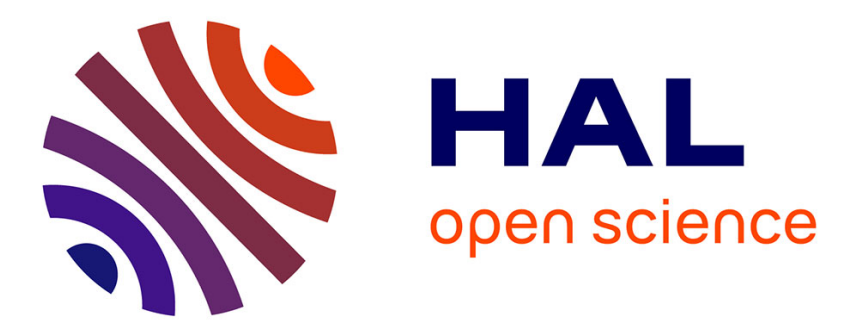

\title{
Mean BER minimization loading algorithm for linear precoded OFDM
}

Fahad Syed Muhammad, Antoine Stephan, Jean-Yves Baudais, Jean-François Hélard

\section{- To cite this version:}

Fahad Syed Muhammad, Antoine Stephan, Jean-Yves Baudais, Jean-François Hélard. Mean BER minimization loading algorithm for linear precoded OFDM. IEEE Sarnoff Symposium, Mar 2009, Nassau Inn in Princeton, United States. pp.1-5, 10.1109/SARNOF.2009.4850349 . hal-00421651

\section{HAL Id: hal-00421651 https://hal.science/hal-00421651}

Submitted on 18 Sep 2014

HAL is a multi-disciplinary open access archive for the deposit and dissemination of scientific research documents, whether they are published or not. The documents may come from teaching and research institutions in France or abroad, or from public or private research centers.
L'archive ouverte pluridisciplinaire HAL, est destinée au dépôt et à la diffusion de documents scientifiques de niveau recherche, publiés ou non, émanant des établissements d'enseignement et de recherche français ou étrangers, des laboratoires publics ou privés. 


\title{
Mean BER Minimization Loading Algorithm for Linear Precoded OFDM
}

\author{
Fahad Syed Muhammad*, Antoine Stephan, Jean-Yves Baudais, and Jean-François Hélard \\ Institute of Electronics and Telecommunications of Rennes (IETR), 35043 Rennes Cedex, France \\ Email*: fahad.syed-muhammad@insa-rennes.fr
}

\begin{abstract}
The problem of minimization of mean bit error rate (BER) of a linear precoded orthogonal frequency division multiplexing (LP-OFDM) system is considered. The discrete bit loading algorithms are proposed, which minimize the mean BER of the system for a target bit rate under a peak power constraint. The results are shown for a power line communication system using a well-known multipath channel model. The other, more common, robustness optimization problem is the margin maximization. The obtained results are compared with those of the margin maximization for both OFDM and LP-OFDM schemes. It is shown that the proposed LP-OFDM allocation performs better than the previously published algorithms for poor SNR. Moreover, it is observed that the LP-OFDM allocations have lower computational complexity than the OFDM allocations.
\end{abstract}

\section{INTRODUCTION}

The optimal exploitation of the available resources is one of the most important aspects of modern-day communication systems. The discrete bit loading algorithms offer a pragmatic solution to sub-optimally allocate suitable numbers of bits and transmit powers to different subcarriers in a multicarrier communication system. Under the assumption that channel state information (CSI) can be made available at the transmitter by sending adequate feedback information from the receiver, the channel knowledge is exploited by using adaptive and variable modulation and different levels of transmit power for different subcarriers. In the result, we increase either the capacity or the robustness of the transmission system. Generally, each subcarrier is assigned a suitable energy, driven by the signalto-noise ratio (SNR), and is loaded with a given modulation, such as different modulation orders of quadrature amplitude modulation (QAM). Linear precoded orthogonal frequencydivision multiplexing (LP-OFDM) is based on classical OFDM combined with a precoding component. The idea is to group together a set of subcarriers with the help of precoding sequences. Each resulting set accumulates the energies of all of its subcarriers to achieve an equivalent SNR such that the total number of bits supported is greater than the sum of the bits supported by each subcarrier individually.

The role of resource allocation in multicarrier communication is to optimize either the throughput or the robustness of the system. Under power spectral density (PSD) constraint and for a given error rate, the resource allocation generally gives either the maximum bit rate for a given system margin or the maximum system margin for a target bit rate. The former is a rate maximization (RM) problem and the latter one is a margin maximization (MM) optimization problem, which is also known in the literature as the problem of power minimization under fixed bit and error rate. The number of bits that can be carried by a given subcarrier, for a given SNR and symbol error rate (SER), is given as [1]

$$
b=\log _{2}\left(1+\frac{S N R}{\gamma \Gamma}\right),
$$

where $\gamma$ is the system margin and $\Gamma$ is the SNR gap presenting the required power difference between the theoretical capacity and the actual rate $b$ conveyed by the selected modulation scheme, while respecting a predefined maximum error rate constraint. For uncoded QAM with a target SER, $P^{s}$, and for a null system margin, $\Gamma$ can be given as [1]

$$
\Gamma=\frac{1}{3}\left[Q^{-1}\left(\frac{P^{s}}{4}\right)\right]^{2},
$$

where $Q^{-1}$ is the inverse of the well-known Q-function.

Classically, to achieve a target error rate, SER is fixed on each subcarrier which is equal to the global SER of an OFDM symbol, since all the constellation sizes of QAM have the same value of the SNR gap at the same SER, as it is clear from (2). In this approach, an approximate relation between SER and bit error rate (BER) is used [2] to achieve the target BER of the system, which results in the violation of the target BER in some cases as discussed in [3]. One solution is to fix the BER rather than SER on each subcarrier as proposed in [4]. Here, we will consider another approach, where rather than fixing the BER at each subcarrier, target BER of a complete OFDM symbol will be achieved, hereafter known as mean BER. This approach has been discussed in [3] and [5].

In this paper, we propose discrete loading algorithms for OFDM and LP-OFDM systems under PSD constraint, where the mean BER minimization (MBM) of the system is performed for a given bit rate. The results are compared with the existing allocation algorithms for both the systems, and it is shown that the proposed algorithms perform better than existing solutions under difficult conditions i.e. poor values of average SNR. It is also observed that the proposed bit loading algorithm for LP-OFDM is much faster than OFDM algorithms. The uncoded QAM is selected as the modulation scheme. This work may also be extended to the study of the coded systems and it might be a promising extension in the future.

The rest of the paper is organized as follows. In Section II, the structure of an LP-OFDM system is described. Section 


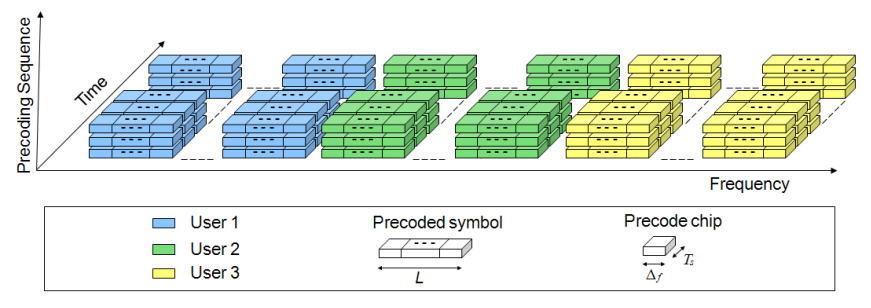

Fig. 1. LP-OFDM system description

III gives the proposed bit loading algorithm for an OFDM system. In Section IV, the bit loading algorithm for an LPOFDM system is devised. In Section V, simulation scenarios are discussed and results are presented for both the systems using a multipath PLC channel model. LP-OFDM and OFDM simulations are run for various average SNRs and different target bit rate, while using the PLC channel model suggested in [6]. Finally, Section VI concludes the paper.

\section{SySTEM DESCRIPTION}

A general LP-OFDM scheme is based on classical OFDM combined with a linear precoding component. The resulting LP-OFDM, whose precoding is applied in the frequency dimension, is known as spread-spectrum multicarrier multipleaccess (SS-MC-MA) in mobile radio communications [7]. In practice, the system is modified by simply adding a precoding block in the transmission chain, thus the system complexity is not significantly increased. Furthermore, the linear precoded component can be exploited to reduce the peak-to-average power ratio (PAPR) of the OFDM system [8]. The linear precoding component improves the signal robustness against frequency selectivity and narrowband interference, since the signal bandwidth could become much larger than the coherence and interference bandwidths. The main advantage of linear precoding in our approach is energy merging by grouping different subcarriers together, which is also useful in increasing the throughput especially under PSD constraint.

The studied LP-OFDM system is shown in Fig. 1. The entire bandwidth is divided into $N$ parallel subcarriers which are split up into $K$ blocks $S_{k}$ of $L$ subcarriers, where $k$ signifies the block number. The precoding function is then applied block-wise by mean of precoding sequences of length $L$. Note that the subcarriers in a given block are not necessarily adjacent. In multiuser context, each user $u$ of the network is being assigned a group $B_{u}$ of subsets $S_{k}$. We emphasize that $\forall u, B_{u}$ are mutually exclusive subsets. Consequently, multiple access between the $U$ users is managed following a frequency division multiple access (FDMA) approach, instead of a code division multiple access (CDMA) approach. It is worthy to mention here that we are only going to consider the single user case. The number of precoding sequences used to spread information symbols on one subset $S_{k}$ is denoted by $C^{(k)}$, with $0 \leq C^{(k)} \leq L$, since we assume orthogonal sequences. A certain amount of energy $E_{c}^{(k)}$ is assigned to each precoding sequence $c^{(k)}$ associated to a given modulation symbol of $b_{c}^{(k)}$ bits, where $1 \leq c \leq C^{(k)}$.

\section{PRoposed OFDM Allocation}

The mean BER of an OFDM symbol is given as [3]

$$
\bar{P}=\frac{\sum_{i=1}^{N} P_{i}^{b} \cdot b_{i}}{\sum_{i=1}^{N} b_{i}},
$$

where $P_{i}^{b}$ and $b_{i}$ are the BER and number of bits supported by subcarrier $i$, respectively. BER is obtained from SER using the approximation $P_{i}^{b} \approx P_{i}^{s} / b_{i}$ [3]. Using this approximation, the optimization problem can be given as

$$
\min \sum_{i=1}^{N} P_{i}^{s} / R
$$

$$
\text { subject to } E_{i} \leq E_{\mathrm{PSD}}, \quad \text { and } R=\sum_{i=1}^{N} b_{i},
$$

where $E_{i}$ is the transmit power of subcarrier $i, E_{\mathrm{PSD}}$ is the defined PSD limit and $R$ is the target bit rate. The objective is to minimize the mean BER of the system for given constraints. From (4) and (3), mean BER directly depends upon SER without considering the number of bits per symbol, therefore SER is used in the proposed algorithm. We consider a multicarrier system employing uncoded M-QAM constellations. For uncoded QAM, SER is given as [9]

$$
P_{i}^{s} \approx 4 Q\left(\sqrt{\frac{3 \mathrm{SNR}_{i}}{M_{i}-1}}\right),
$$

where $\mathrm{SNR}_{i}$ and $M_{i}$ are the SNR value and the constellation assigned to subcarrier $i$, respectively. Here, we propose an iterative algorithm which allocates bits to the subcarrier, which has the best SER (i.e. the minimum value of $P_{i}^{s}$ ). It should be noted that all the subcarriers use all the available transmit power no matter what is the constellation size. The algorithm can be described as follows.

$$
\begin{aligned}
& \text { 1: Initiate all the subcarriers with zero bit } \\
& \text { 2: while } \sum_{i=1}^{N} b_{i}<R \text { do } \\
& \text { 3: } \quad i=\arg \min _{i} P_{i}^{s}\left(b_{i}\right) \\
& \text { 4: } \quad b_{i}=b_{i}+1 \\
& \text { 5: end while }
\end{aligned}
$$

The allocation provided by this algorithm is similar to the one obtained from optimal margin maximization solution. In the next section, we will consider resource allocation of LPOFDM systems.

\section{Proposed LP-OFDM AllocAtion}

As explained earlier, an LP-OFDM system groups together different subcarriers into block of subcarriers. Here, firstly we will study a mono block LP-OFDM system and will devise discrete bit loading algorithm, which minimizes the mean BER 
of the system for a given PSD mask and a target bit rate. This approach will further be extended for a multi block LP-OFDM system.

\section{A. Single block LP-OFDM}

The MBM problem for a single block LP-OFDM system can be described as follows

$$
\begin{gathered}
\min \sum_{c=1}^{C} P_{c}^{s} / R, \\
\text { subject to } \sum_{c=1}^{C} E_{c} \leq E_{\mathrm{PSD}}, \text { and } \sum_{c=1}^{C} b_{c}=R,
\end{gathered}
$$

where $C$ is the number of useful precoding sequences in a block, $P_{c}^{s}, b_{c}$ and $E_{c}$ are the SER, number of bits and the transmit power associated with the precoding sequence $c$, respectively. $E_{\mathrm{PSD}}$ is the defined PSD limit and $R$ is the target bit rate of the considered block. SER of the precoding sequence $s$, in a given block can be written as

$$
P_{c}^{s}=2 \operatorname{erfc}\left(\sqrt{\frac{\alpha E_{c}}{2^{b_{c}}-1}}\right)
$$

where $\alpha$ is given as

$$
\alpha=\frac{3}{2} \frac{L^{2}}{\sum_{i=1}^{L} \frac{1}{\left|H_{i}\right|^{2}}} \frac{1}{N_{0}},
$$

where $H_{i}$ is the frequency response of subcarrier $i$, and $N_{0}$ is the noise level. We find, after analytically solving the optimization problem, that the optimal solution is to equally distribute the bits and power among all the precoding sequences. The next step is to find the optimal number of useful precoding sequences $C^{*}$. This step was performed by linear regression using (6) and (7), and taking $E_{c}=E_{\mathrm{PSD}} / C^{*}$ and $b_{c}=R / C^{*}$. The obtained solution is given as

$$
C^{*}=\operatorname{round}\left(m\left(e^{\frac{R-0.42}{1.44}}-1\right)\right),
$$

where $m$ is given as

$$
m=\frac{2 \alpha E_{\mathrm{PSD}}}{3\left(2^{R}-1\right)} .
$$

The proposed pragmatic solution is the discrete bit loading algorithm, where for a given target bit rate and a given block length, $L$, we find the optimal number of useful precoding sequences. In the case of integer bit allocation, the optimal bits distribution, for an optimal number of useful precoding sequences, $C^{*}$, would be to allocate $\left\lfloor R / C^{*}\right\rfloor+1$ bits to $n$ precoding sequences and $\left\lfloor R / C^{*}\right\rfloor$ bits to the remaining $C^{*}-n$ precoding sequences. Since we have a target throughput $R, \mathrm{n}$ should respect the equation $n\left(\left\lfloor R / C^{*}\right\rfloor+1\right)+$ $\left(C^{*}-n\right)\left\lfloor R / C^{*}\right\rfloor=R$. Thus, we find

$$
n=R-C^{*}\left\lfloor R / C^{*}\right\rfloor \text {. }
$$

Consequently, the integer bit allocation solution for a singleblock system is given by

$$
\begin{cases}b_{c}=\left\lfloor R / C^{*}\right\rfloor+1, & \forall c \in[1: n] \\ b_{c}=\left\lfloor R / C^{*}\right\rfloor, & \forall c \in\left[n+1: C^{*}\right] .\end{cases}
$$

After deciding the suitable number of bit for all the precoding sequences, the transmit power is divided among the precoding sequences in the following manner,

$$
E_{c}=\frac{\left(2^{b_{c}}-1\right)}{\sum_{c=1}^{C^{*}}\left(2^{b_{c}}-1\right)} E_{\mathrm{PSD}} .
$$

The algorithm can be described as follows.

1: Compute $C^{*}$ from (9)

2: if $C^{*}<1$ then

3: $\quad C^{*}=1$

4: else if $C^{*}>L$ then

5: $\quad C^{*}=L$

6: end if

7: Calculate $n$ from (11)

8: Find $b_{c}$ from (12)

9: Find $E_{c}$ from (13)

This algorithm performs a faster allocation of bits and transmit power and performs better than OFDM allocation and $\mathrm{MM}$ allocation under difficult conditions. It also has significantly low computational complexity, as no iterative procedure is used.

\section{B. Multi block LP-OFDM}

The MBM optimization problem for a Multi block LPOFDM system can be given as

$$
\min \frac{\sum_{k=1}^{K} P_{k}^{b} R_{k}}{\sum_{k=1}^{K} R_{k}},
$$

$$
\text { subject to } \sum_{k=1}^{K} R_{k}=R, \text { and } \sum_{c=1}^{C} E_{c} \leq E_{\mathrm{PSD}}, c \in S_{k},
$$

where $P_{k}^{b}$ is the mean BER of block $k$. Contrary to the proposed algorithm for OFDM, which uses SER per subcarrier, we can not use SER per block because $P_{k}^{b} R_{k}$ does not define the SER of the given block. Here, we propose an iterative algorithm which allocates bits to the block, which has the best mean BER (i.e. the minimum value of $P_{k}^{b}$ ). Similar to the case of OFDM, here all the blocks use all the available transmit power no matter what is the constellation size. In this manner, we find the target bit rate of each block. The second step consists in finding the optimal number $b_{c}$ of bits that should be allocated to each precoding sequence in order to achieve the block throughput $R_{k}$, as explained in Section IV-A. The algorithm can be described as follows.

$$
\text { 1: while } \sum_{k=1}^{K} R_{k}<R \text { do }
$$




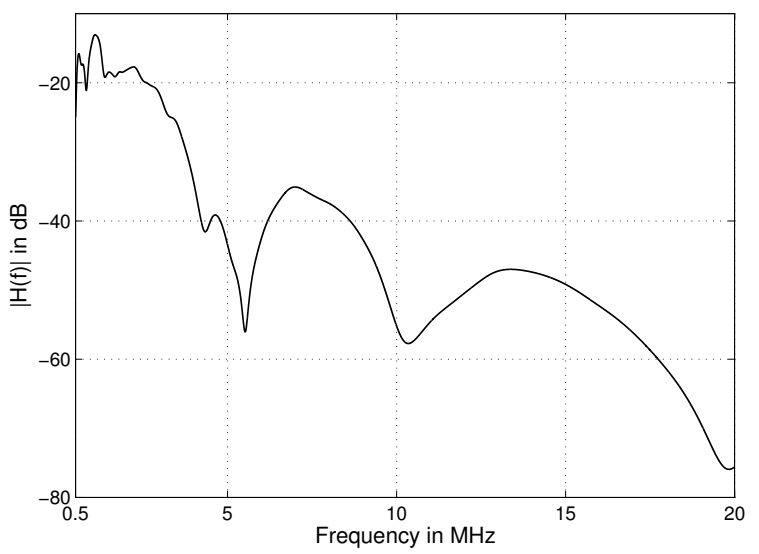

Fig. 2. 15-paths reference channel model for PLC [6]

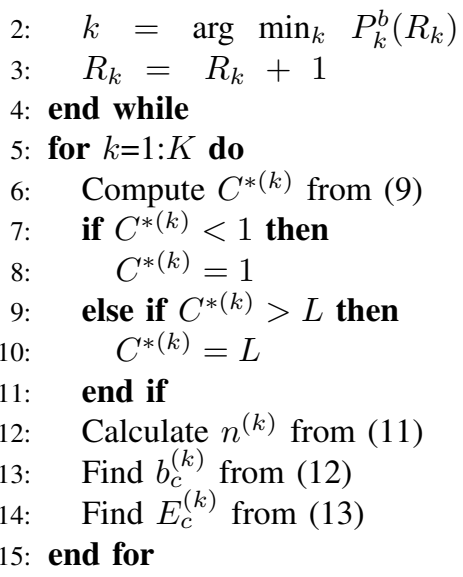

This algorithm is also faster than that of OFDM, as the number of possible decisions has decreased in the first while loop because we have grouped together multiple subcarriers.

\section{Simulations And Results}

In this section, we will present simulation results, comparing the performances of $\mathrm{MM}$ and $\mathrm{MBM}$ allocations. We use the MM allocations proposed in [10], for both OFDM and LPOFDM scenarios. In [10], the proposed allocation for LPOFDM was generalized for OFDM by taking $L=1$. To compare the results of the proposed allocations with the MM allocations, we calculated the mean BER, by using (4), (6) or (14), depending upon the suitable configuration, for all the algorithms after finalizing the bit distribution. The generated signal is a baseband signal produced by dividing $20 \mathrm{MHz}$ band of Zimmermann channel into 1024 subcarriers. The maximum number of bits per symbol is limited to 15 and the value of $L$ for LP-OFDM systems is taken as 32 . It is assumed that the synchronization and channel estimation tasks have been successfully performed. We use the multipath model for the power line channel, proposed in [6] and presented in Fig. 2. The considered reference model is $110 \mathrm{~m}$ link 15-paths model
TABLE I

PARAMETERS OF THE 15-PATH MODEL

\begin{tabular}{|c|c|c|c|c|c|}
\hline \multicolumn{6}{|c|}{ attenuation parameters } \\
\hline \multicolumn{2}{|r|}{$k=1$} & \multicolumn{2}{|c|}{$a_{0}=0$} & \multicolumn{2}{|c|}{$a_{1}=2.5 \cdot 10^{-9}$} \\
\hline \multicolumn{6}{|c|}{ path-parameters } \\
\hline$i$ & $g_{i}$ & $d_{i}(\mathrm{~m})$ & $i$ & $g_{i}$ & $d_{i}(\mathrm{~m})$ \\
\hline 1 & 0.029 & 90 & 9 & 0.071 & 411 \\
\hline 2 & 0.043 & 102 & 10 & -0.035 & 490 \\
\hline 3 & 0.103 & 113 & 11 & 0.065 & 567 \\
\hline 4 & -0.058 & 143 & 12 & -0.055 & 740 \\
\hline 5 & -0.045 & 148 & 13 & 0.042 & 960 \\
\hline 6 & -0.040 & 200 & 14 & -0.059 & 1130 \\
\hline 7 & 0.038 & 260 & 15 & 0.049 & 1250 \\
\hline 8 & -0.038 & 322 & & & \\
\hline
\end{tabular}

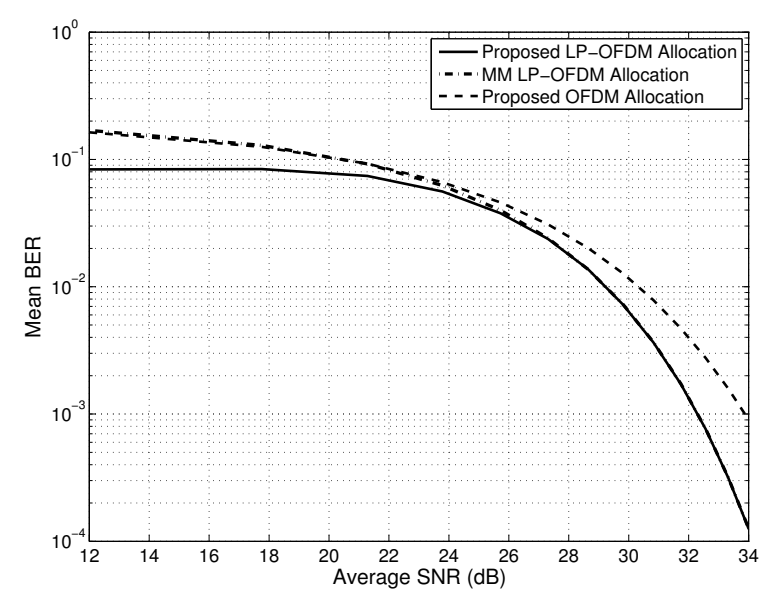

Fig. 3. Mean BER comparison of the proposed allocations with Margin Maximization allocations for different Average SNR, for $R=4000 \mathrm{bit} / \mathrm{OFDM}$ symbol.

whose frequency response is given by

$$
H(f)=\sum_{i=1}^{N} g_{i} \cdot e^{-\left(a_{0}+a_{1} f^{k}\right)^{d_{i}}} \cdot e^{-j 2 \pi f d_{i} / v_{p}},
$$

where $v_{p}$ depends upon the dielectric constant of the electric line. The parameters of the 15-path model are listed in Table I, and $\tau_{i}$ is the delay of path $i$. A background noise level of $-110 \mathrm{dBm} / \mathrm{Hz}$ is assumed and the signal is transmitted with respect to a flat PSD of $-40 \mathrm{dBm} / \mathrm{Hz}$.

Fig. 3 shows the achieved mean BER versus the average SNR at a fixed target bit rate of $4000 \mathrm{bit} / \mathrm{OFDM}$ symbol. The performance of the proposed allocations for OFDM and LPOFDM is compared with the MM allocation for LP-OFDM, as the performance of the proposed OFDM system is similar to that of MM OFDM allocation. The LP-OFDM system is not generalized to get an OFDM system for $L=1$, as it 


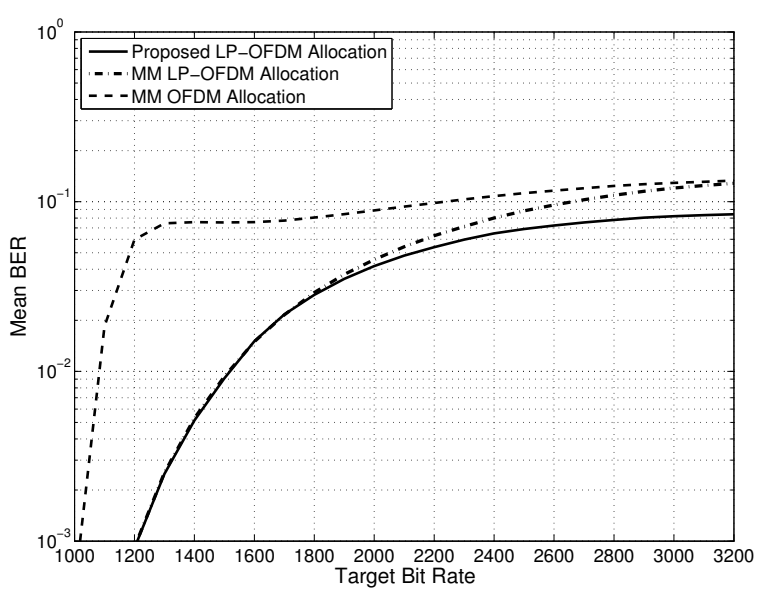

Fig. 4. Mean BER comparison of the proposed allocations with Margin Maximization allocations for various bit rates at average $\mathrm{SNR}=12 \mathrm{~dB}$

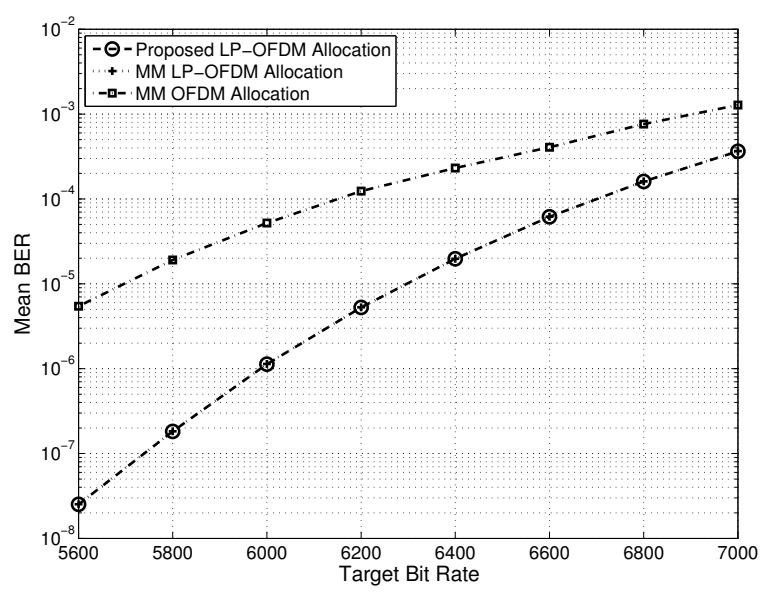

Fig. 5. Mean BER comparison of the proposed allocations with Margin Maximization allocations for various bit rates at average $\mathrm{SNR}=43.78 \mathrm{~dB}$

considers the BER of a block. While in the case of OFDM system, it is optimal to consider the SER of a subcarrier. Furthermore, it is shown that the performance of the proposed LP-OFDM solution is better than MM allocations for both OFDM and LP-OFDM solutions, especially for lower SNRs. At higher SNRs, both LP-OFDM allocations perform equally well, but still the proposed LP-OFDM allocation is better than that of OFDM. The proposed LP-OFDM performs better than $\mathrm{MM}$ allocation at lower average SNR, as it uses the optimal number of useful precoding sequences, while the MM LPOFDM allocation always uses all the precoding sequences of a block i.e. $L$. For higher average SNR, the calculated optimal value of useful precoding sequence $C^{*}$ is equal to $L$. It might be further optimized by using the optimal block length, which might be another promising extension in the future.

Fig. 4 compares the performance of different allocations for different values of target bit rates at a low value of average SNR (i.e. $12 \mathrm{~dB}$ ). The results show that the proposed LPOFDM allocation performs better than MM LP-OFDM and OFDM allocations. Similarly, Fig. 5 compares the performance of all considered allocations for different values of target bit rates at a high value of average SNR (i.e. $43.78 \mathrm{~dB}$ ). It is shown that the performances of LP-OFDM allocations are comparable but the LP-OFDM solution performs better than the OFDM one. It shows that the proposed system is more useful at poor channel conditions.

\section{CONCLUSiON}

In this paper, we propose bit allocation algorithms for OFDM and LP-OFDM systems, which minimize the mean BER of the system for a fixed target bit rate and a given PSD mask. These algorithms provide better results than the MM allocations, while both are serving the purpose of robustness maximization of the system. Furthermore, the performance of LP-OFDM is better than that of OFDM system, because it offers different numbers of useful precoding sequences as an additional degree of freedom for optimization. It is shown that the proposed allocation for LP-OFDM performs better at lower average SNR and for higher target bit rates. The complexity of the LP-OFDM is also reduced with respect to that of OFDM, as the number of possible decisions decreases by grouping different subcarriers.

\section{REFERENCES}

[1] J. Cioffi, A multicarrier primer ANSI T1E1.4/91-157, 1991, Committee contribution, Tech. Rep.

[2] T.N. Zogakis, J.T. Aslanis, and J.M. Cioffi, "A Coded and shaped discrete multitone system,” IEEE Trans. Commun., vol. 43, no. 12, pp. 2941-2949, Dec. 1995.

[3] A. M. Wyglinski, F. Labeau, and P. Kabal, "Bit loading with BERconstraint for multicarrier systems," IEEE Trans. Wireless Commun., vol. 4, no. 4, pp. 1383-1387, Jul. 2005.

[4] F. Syed-Muhammad, J.-Y. Baudais, J.-F. Hélard, and M. Crussière, "A coded bit-loading linear precoded discrete multitone solution for power line communication," in Proc IEEE Workshop on Signal Processing Advances in Wireless Commun., Recife, Brazil, 2008, pp. 555-559.

[5] Y. George, and O. Amrani, "Bit Loading Algorithms for OFDM," in Proc IEEE Inter. Symp. on Info. Theory., Chicago, USA, 2004, pp. 391.

[6] M. Zimmermann and K. Dostert, "A multipath model for the powerline channel," IEEE Trans. Commun., vol. 50, no. 4, pp. 553, Apr. 2002.

[7] K. Fazel, and S. Kaiser, Multi-Carrier and Spread Spectrum Systems, John Wiley \& Sons, 2003.

[8] S. Nobilet, J.-F. Hélard, and D. Mottier, "Spreading sequences for uplink and downlink MC-CDMA systems: PAPR and MAI minimization," European Trans. on Telecomm., vol. 13, no. 5, pp. 465-474, 2002.

[9] J. G. Proakis, Digital Communications, 4th ed. New York: McGraw-Hill, 2000.

[10] M. Crussière, J.-Y. Baudais, and J.-F. Hélard, "Loading Algorithms for Adaptive SS-MC-MA Systems over Wireline Channels: Comparison with DMT," European Transactions on Telecommunications, Special Issue on the 5th Multi-Carrier Spread-Spectrum vol. 17, no. 6, pp. 659-669, 2006.

[11] I. Kalet, "The Multitone Channel," IEEE Trans. Commun., vol. 37, no. 2, pp. 119-124, Feb 1989.

[12] J. Boutros, and E. Viterbo, "Signal space diversity: a power- and bandwidth-efficient diversity technique for the Rayleigh fading channel," IEEE Trans. Inform. Theory, vol. 44, pp. 1453-1467, Jul 1998.

[13] D. Rainish, "Diversity transform for fading channels," IEEE Trans. Commun., vol. 44, pp. 1653-1661, Dec. 1996.

[14] Z. Wang, and G. B. Giannakis, "Linearly precoded or coded OFDM against wireless channel fades?," in Proc IEEE Workshop on Signal Processing Advances in Wireless Commun., Taoyuan, Taiwan, 2001, pp. 267-270. 